Témoigner Témoigner. Entre histoire et mémoire

Getuigen Revue pluridisciplinaire de la Fondation Auschwitz

$121 \mid 2015$

Violences radicales en scène

\title{
Michel Dufranne, Milorad Vicanović, Christian Lerolle, Triangle rose
}

Tournai, Quadrants, 2011

\section{Yoann Sarrat}

\section{(2) OpenEdition}

Journals

Édition électronique

URL : https://journals.openedition.org/temoigner/3727

DOI : $10.4000 /$ temoigner.3727

ISSN : 2506-6390

Éditeur :

Éditions du Centre d'études et de documentation Mémoire d'Auschwitz, Éditions Kimé

Édition imprimée

Date de publication : 1 octobre 2015

Pagination : 212-213

ISSN : 2031-4183

Référence électronique

Yoann Sarrat, « Michel Dufranne, Milorad Vicanović, Christian Lerolle, Triangle rose », Témoigner. Entre histoire et mémoire [En ligne], 121 | 2015, mis en ligne le 01 octobre 2016, consulté le 04 février 2022. URL : http://journals.openedition.org/temoigner/3727 ; DOI : https://doi.org/10.4000/temoigner.3727

Ce document a été généré automatiquement le 4 février 2022

Tous droits réservés 


\section{Michel Dufranne, Milorad Vicanović, Christian Lerolle, Triangle}

\section{rose}

Tournai, Quadrants, 2011

\section{Yoann Sarrat}

\section{RÉFÉRENCE}

Michel Dufranne, Milorad Vicanović, Christian Lerolle, Triangle rose, Tournai, Quadrants, 2011, 146 p.

1 Le « Paragraphe 175 » est un article du Code pénal allemand actif de 1871 à 1994 . C'est au nom de cette loi que les homosexuels seront persécutés, déportés et exterminés pendant la Seconde Guerre mondiale.

2 La bande dessinée de Michel Dufranne, scénariste, et Milorad Vicanović, dessinateur, Triangle Rose, parue en 2011 aux éditions Quadrants Astrolabe, raconte l'histoire d'un survivant à travers plusieurs figures importantes. Elle démarre sur ce que nous pourrions qualifier maintenant de topos contemporain d'un groupe de lycéens devant remettre un devoir sur la Shoah, mais plutôt que de bâcler l'exercice en recopiant des pages de Wikipédia comme d'autres l'ont prévu, un jeune homme, qui a « un plan » (p.3), décide de rendre visite à son arrière-grand-père qui, apparemment, " y a survécu » (p. 3) : «mon père m'a juste dit que s'il était pas sympa et ressemblait à un vampire, c'est parce qu'il avait survécu aux camps... » (p. 7)

3 Par la suite, l'histoire des «Triangles roses » est vue à travers les yeux de ce témoin, Andreas, qui apparaît comme aigri et méchant aux trois lycéens venus s'aventurer dans sa demeure : "c'est un travail sur... La Deuxième Guerre mondiale ", " Seconde ! On dit Seconde Guerre mondiale quand on est optimiste !!» (p. 10) Pour lui, il y a l'avant, le pendant et l'après (les " années de larmes »). Ce dernier étant aussi difficile que le précédent, parce qu'il faut le rappeler: les homosexuels, qui n'étaient pas reconnus 
comme victimes, ne recevaient aucune indemnisation, ils continuaient également d'être persécutés, y compris par d'autres survivants qui voyaient en eux des voleurs de pain. « Où vivez-vous quand la guerre a commencé ?", «Berlin », «à Berlin ?! », « oui, Berlin! à l'époque j'étais allemand», «Allemand ?! et tu t'es quand même retrouvé dans les camps ?? » (p. 12)

4 L'avant, c'est le Berlin de l'entre-deux-guerres, les "années brunes », longuement racontées dans le chapitre premier, dans lequel le Paragraphe 175 est désuet dans une ville particulièrement culturelle et ouverte, les gays et lesbiennes se réunissant librement dans plusieurs clubs, des personnes intelligentes, cultivées, discutant, comme d'autres, de l'actualité, de la politique, du nazisme. Mais c'est aussi la montée en puissance des nazis et, dès le début particulièrement brutal, de la persécution : les homosexuels sont traqués, poursuivis, tabassés, etc. Dénoncé par la concierge de son immeuble, le personnage principal est emprisonné avec d'autres détenus violents, la police nazie lui propose plusieurs arrangements, dont une dénonciation de ses amis contre une cellule en isolement puis une castration, pour être libéré.

5 Le pendant, c'est le camp, les " années noires ", rapidement, mais brutalement rendues par les dessins de Vicanović et l'écriture de Dufranne, ce qui constitue un choix stylistique et formel impressionnant, dans le sens où le lecteur est saisi, voire submergé par ces images atroces et nombreuses. Parmi celles-ci, la «Singing Forest»: les homosexuels, qui portaient un triangle rose sur leurs uniformes - ce qui constituait une seconde discrimination intraconcentrationnaire -, étaient suspendus par les bras, vers l'arrière, à des troncs d'arbres lors de punitions. Dans le très beau documentaire de Rob Epstein, Paragraph 175, les cris de ces suppliciés, inhumains, sont souvent décrits par les témoins. La survie était donc particulièrement difficile pour ces détenus.

6 Un autre procédé, bien que topique, utilisé dans l'œuvre, est à noter. Il concerne le remarquable travail de couleurs de Milorad Vicanović et Christian Lerolle: les souvenirs relatifs à l'horreur nazie sont dessinés en noir et blanc, seules les diverses étoiles que devaient porter les déportés sont colorées et ressortent intensément de la page, devenant des objets allégoriques (procédé que l'on retrouve notamment, dans une autre mesure, dans des films comme La Liste de Schindler).

7 La bande dessinée et le film montrent quelques visages et corps d'êtres marqués par cette expérience douloureuse. Ce qui ressort, d'ailleurs, c'est que certains ont d'immenses difficultés à témoigner, d'autres refusent complètement, mais tous sont d'accord pour dire que le devoir de mémoire n'a pas fonctionné pour eux. Triangle Rose rappelle que les persécutions ont continué jusqu'en 1995, y compris pendant les événements commémoratifs. Le dernier et court chapitre se nomme: "devoir de mémoire (imprimé en noir en haut à gauche) / droit à l'oubli (imprimé en blanc en bas à droite) ", une antithèse brutale sur une pleine page impliquant la silhouette sombre du narrateur sur la tempe duquel un revolver est pointé et apparaissant juste après l'écoute du discours de Jacques Chirac du 24 avril 2005 : « en Allemagne, mais aussi sur notre territoire, celles et ceux que leur vie personnelle distinguait, je pense aux homosexuels, étaient poursuivis, arrêtés et déportés. Aujourd'hui nous savons... », et au personnage d'éteindre la télévision, puis de compléter : « ... que tout ceci arrive bien trop tard... » (p. 140)

8 Ce chapitre conclusif se lance sur les larmes, commençant à couler dans la dernière case de l'introduction, de ce vieux monsieur, et nous découvrons alors qu'il n'a rien raconté aux lycéens : « on ne vous apprend vraiment rien à l'école... Les Allemands ont 
été les premiers dans les camps! Ils les ont inaugurés voire construits pour certains !! Renseignez-vous avant de venir importuner un vieil homme... c'est quand même les moindre des politesses non ?! Rentrez chez vous et trouvez-vous de bons livres. Tout est déjà dans les livres...» (p. 142), la bande dessinée s'achève alors sur cette décision : «ton arrière-grand-père, c'est vraiment un gros connard! [...] on va chez moi et on "pipotte" un truc avec Wiki? » (p.143), ainsi que sur la solitude d'un homme accablé par les souvenirs ne parvenant pas à raconter ni à témoigner.

9 Le corpus d'œuvres traitant de cette terrible période et de cette horrible condition reste assez faible, mais il faut notamment lire l'impressionnant et poignant témoignage de Pierre Seel, également présent dans le film d'Epstein: Moi Pierre Seel, déporté homosexuel, qui raconte cet avant, ce pendant et cet après douloureux. Nous renvoyons aussi à l'essai, très riche et complet, de Jean Le Bitoux, qui s'intitule Les oubliés de la mémoire. Par ailleurs, un film expérimental, Il Rosa Nudo (Le Rose $\mathrm{Nu}$ ), réalisé par Giovanni Coda, basé sur la vie de Pierre Seel, est sorti en 2013. 